\title{
Linguistic evidence for a Lao perspective on facial expression of emotion ${ }^{1}$
}

\author{
N. J. Enfield
}

In the ongoing debate about emotions and their relationship with facial expression, James Russell has recently campaigned for "the gathering of new evidence" (1995: 382) concerning the nature and meaning of facial expressions across cultures and across languages. In this paper, I present data on some ways in which Lao people describe facial expression, as well as some of the ways they attribute inner states to people making particular facial displays. I first discuss briefly a number of words and expressions available in Lao for the description of emotions and other inner states. In the second section, I describe some of the ways in which Lao speakers isolate, recognise and describe particular facial expressions, as well as making some comments on their attribution of inner states (of thought, feeling, and/or emotion) to people making the expressions in question. One point I want to make is that "folk" analyses of semiotic phenomena, revealed in linguistic semantics, are fundamental to a well-informed comparative science of emotion and nonverbal communication.

\section{1. "Emotions" in Lao}

There are no exact equivalents in Lao of the English words sad, angry, disgusted, happy, surprised, and afraid. In some cases, there are obvious rough translations of these so-called "basic emotions" (Ekman 1992), while in others the equivalents are not so clear. Let me introduce some of the problems.

\section{1. 'Sad'}

There are several possible translations into Lao of the English word sad. The most likely to correspond to the canonical sense of English sad is sia- 
caj, literally 'lost-heart'. Sia-caj shares with sad the idea of bad feelings as a response to some unfortunate event. Thus, one can be sia-caj 'about' something, or sia-caj 'that' something has happened, apparently restricted to cases in which something happens to the experiencer. For example, a speaker says he was sia-caj when, having hurried home from the market to get money to buy a bargain-priced turkey, returns to find that the creature has already been sold.

Another common term often translatable as sad is sook-saw, which is more likely to carry the connotation of a longer term state of unhappiness, due to some sorry state the subject finds themselves in, rather than some specific event. A third term which overlaps to some extent with sad is ngaw, which most typically expresses a bad feeling of loneliness or aloneness, especially when a person is too often by themselves in overly quiet surroundings. The feeling of $n g a w$ is no doubt related to the premium in Lao culture on muan 'fun', and being with тии 'friends, the group'. Lao people are most comfortable spending time in larger groups of people with plenty of activity going on. Feeling $n g a w$ is a response to aloneness in this cultural context.

\section{2. 'Angry'}

There are two expressions in Lao which can be routinely translated, in various contexts, by the English word angry. The first is caj-haaj 'offensiveheart' (in the sense of 'heart-on-the-offensive'), which expresses the emotional state of a person who is responding to some incident which directly and negatively affects them. Someone who is caj-haaj is preferably avoided, since they would be likely to say (or perhaps do) something bad to anyone who they may consider responsible for whatever it is they are reacting to. In this sense, caj-haaj is quite like English angry, but the two differ in that caj-haaj cannot usually be used to express an idea of being angry about some situation which does not directly affect the person involved. It is more immediate. (Note that the component haaj may be used as a speech-act verb - to 'tell somebody off' - and Lao speakers normally find it upsetting, as recipient or bystander, when this behaviour occurs.)

The second common 'anger' term is khiat, where the negative feeling is typically not overtly expressed, similar in some contexts to Australian English pissed off. In contrast with caj-haaj, it is non-expression which char- 
acterises a typical state of being khiat. If someone is khiat with/at someone else, they are likely not to speak to that person, nor to do anything with or for that person. In the kinds of close-knit and cooperative village settings typical in Lao society, to ignore someone is to send a strong signal.

The idea of khiat as 'non-confrontational anger' relates to a strong value in Lao culture of avoiding overt confrontation (especially the possibility of people "saying things") where possible. A key expression encapsulating this is the ubiquitous bò-pen-ñang [not be anything] 'no problem, it's nothing', which epitomises a core ideal that one should tackle problems with a degree of acceptance and tolerance, and without kò̀-lùang [build-issue] 'creating an issue'. If Lao people are 'angry' at each other, they are not as likely to make their bad feelings explicitly known in confrontational ways, but will rather "express" them through, literally, non-expression. This remains an effective way of getting the message across.

\section{3. 'Disgusted'}

There is a fairly clear translation into Lao of English disgusted, namely khidiat. The prefix $k h i$ - (related to khii 'shit') is used widely in the language for a range of idiomatic expressions to do mainly with different kinds of effluent material and waste products ( $k h i-m u u k$ [muuk $=$ nose] 'mucus'; $k h i$ thaw [thaw = 'ash'] 'ashes'; khi-lùaj [lùaj = 'mill'] 'sawdust'), as well as negative personal characterisations ( $k h i-k h u j[k h u j=$ 'chat'] 'snobby'; $k h i$ lak [lak = 'steal'] 'thief'; khi-qaaj [qaaj = 'shy'] 'overly shy'). (The morpheme -diat has no independent meaning outside of the expression khi-diat 'disgusted'.) The usage of khi-diat is more restricted than that of the English term disgusted, since the Lao term is only used to refer to responses evoked by things which are literally physically disgusting, such as the idea of eating certain things, or of dealing with physically revolting things such as rotten food, etc. In Lao, for example, one cannot be disgusted 'at' someone, or 'that' something has happened.'

\section{4. 'Happy'}

The English expression happy may be translated into Lao as dii-caj 'goodheart', sabaaj(-caj) 'content(-heart)' or mii khuam-suk 'have contented- 
ness'. Dii-caj has a close affinity with the English expression glad, being a transient positive emotional response to some specific fortunate incident. Thus, one may be dii-caj 'that' something has happened, but unlike English happy, dii-caj cannot describe a feeling of general contentedness (as in John is happy with his new job or Mary seems happy these days). This latter idea is partly covered by the term sabaaj 'content/comfortable', a cultural key word for Lao speakers (as for speakers of neighbouring Thai). Sabaaj itself refers to a feeling rather than an emotion, since it does not entail a cognitive component - it often means 'comfortable', for example with regard to seating, clothing, or ambience. However, in a compound with - $c a j$ 'heart', the meaning does include the cognitive evaluation leading to the feeling. This prior cognitive evaluation is the hallmark of emotion (according to many; but cf. e.g. Zajonc 1980, 1984).

Another expression corresponding in certain cases to happy is mii khuam-suk, literally 'to have happiness'. (Khuam 'sense' acts here as a nominalising prefix to the verb-like bound element suk 'content/happy'.) This expression is used in many cases which would also be compatible with sabaaj, but it does not permit reference to exclusively physical comfort which sabaaj 'content' allows. Rather, it is a profound state of wellbeing which normally refers to one's general condition of mind and soul. While one may be dii-caj 'glad' to see an unexpected visitor, it would be odd to mii khuam-suk 'have happiness' in the same way. On the other hand, an aim in life could be to mii khuam-suk 'have happiness', but it would make little sense for one to generally pursue being dii-caj 'glad'.

\section{5. 'Surprised'}

Two Lao translations are possible for English surprised. These are tok-caj 'fall-heart' and tùùn 'awaken'. Both involve a feeling of reaction to the unexpected, but the second entails an instinctive physical reaction (a 'start'), and there is no entailment of any thought or evaluation preceding and/or causing the feeling of 'surprise'. ${ }^{3}$ (Cf. Goddard 1997 on a similar distinction in Malay.) Tok-caj, however, can include the idea of a cognitive evaluation leading to the feeling of 'surprise'. Thus, one can be tok-caj, but not tùùn, 'that' something has happened. This element of evaluation leading to feeling would seem to point to tok-caj as an emotion, and tùùn as a mere feeling or instinctive physical reaction. Even so, the term tok-caj is not pre- 
cisely equivalent to surprise. For example, tok-caj cannot express the kind of 'surprise' expressed in English I'm surprised you're still here. With respect to tok-caj, some specific event must accompany the evaluation leading to the feeling of 'surprise'.

\section{6. 'Afraid'}

The most obvious translation of afraid or scared into Lao is jaan. It is unclear to me what difference exists between Lao jaan and English afraid or scared. It would seem that jaan is closer to afraid in its being less suggestive of immediate and present danger. Jaan is also used in Lao as a complement-taking predicate expressing what the speaker merely suspects rather than literally fears (cf. English I' $m$ afraid she's not coming), and unlike English it need not have a negative connotation (cf. Lao jaan phòo [afraid enough] '(I) suspect (there'll be) enough [e.g., rice to eat]' vs. English ?' $m$ afraid there'll be enough).

\subsection{A note on $c a j$, the Lao 'heart/mind'}

The term caj, which elsewhere refers to the physical 'heart' or 'centre', occurs in most Lao expressions related to emotion, and denotes the primary 'seat' of cognitive and emotional activity for the Lao, the so-called 'heart' or 'mind'. This word is cognate with Thai cay (Diller and Juntanamalaga 1990), and shares many of the same idiomatic and grammatical properties. Both the Lao and Thai terms also show extensive semantic and grammatical similarity with Malay hati and comparable terms in other Southeast Asian languages (cf. Matisoff 1986, Oey 1990, Jaisser 1990 and Goddard this volume - Enfield 2001 provides a methodological and theoretical discussion).

There are dozens of expressions in Lao involving caj, of roughly two main types. A set of terms describing lasting character traits treat $c a j$ as a regular nominal head, with some quasi-adjectival modifier following (e.g. caj-dam [heart-black] 'ruthless', caj-kuang [heart-broad] 'generous', caj$k a a$ [heart-dare] 'daring, courageous'). Most emotion terms, referring to more transient characterisations, place caj second in the combination (e.g. nò̀j-caj [small heart] 'offended', ñaak-caj [difficult-heart] 'undeciding, 
tortured', tok-caj [fall-heart] 'surprised'). There are, however, exceptions to these generalisations (cf. caj-haaj 'angry' $\$ 1.2$., above). The issue of $c a j$ compounds and their grammatical and semantic properties deserves an extensive study.

\subsection{Comment}

We began with the question of correlations across languages between emotions and facial expressions. We are yet to consider facial expressions, but it is already clear that particular facial expressions cannot be said to universally express particular emotions. This is because when eliciting and/or describing Lao speakers' attribution of inner states to people making various facial displays, the researcher cannot investigate ideas such as angry, happy, or sad, since Lao people do not use these English words when speaking Lao, and there is no guarantee that they would understand them if they were used. Instead, the terms used are Lao. And as discussed above, the Lao terms do not have equivalents in English. So, even if we accept that the Lao and English categories are indeed "emotions", they are not the same emotions.

\section{Conventional description of facial expression in Lao}

I now describe some ways of speaking about facial expression in Lao, concentrating on lexical items and idioms which can be used to describe particular facial expressions (or features thereof). I will note the kinds of attributions of inner/emotional states which Lao people make, in their own terms, to people with these facial expressions. I am not concerned here with such questions as the factual accuracy of attribution of a particular emotional state to some facial expression, nor am I interested here in correlation, causal or otherwise, of inner states and facial displays. The aim is to present linguistic evidence for the Lao speaker's conventional analysis of the face. 


\subsection{Grammatical properties of facial expression descriptions in Lao}

While English has a range of simple words for facial expressions (e.g. scowl, grin, smile, grimace), Lao notably has few. The only one attested below is nim 'smile'. Many of the rest are more explicitly descriptive polymorphemic expressions, such as 'rotten face', 'smelly face', 'stuck eyes', and 'clenched mouth'. In between these extremes are a number of 'cranberry' expressions (i.e. in which one component does not independently occur), such as naa-beq 'ready-to-cry face' and taa-loo 'exposed-whitesabove-iris eyes', in which the modifying elements (beq and loo, respectively) have no independent meaning.

Syntactically, these facial expression descriptions involving head nominals such as paak 'mouth', taa 'eye(s)', or naa 'face' may pattern either as (a) noun + predicate, in a kind of relative clause or noun + attribute construction $(\S 2.4 .4$., below):

\section{taa-khaang}

eye-stuck

'stuck eyes'; 'eyes which are stuck';

or (b) a kind of incorporating verb + object construction ( $\$ 2.3 .4$., below):
ñom-paak
clench-mouth
'clench-mouthed'.

\subsection{The face}

The face is referred to in Lao as naa, or baj-naa (where baj elsewhere means 'leaf' and serves as a classifier for flat handle-able things such as sheets of paper). The word naa performs a range of other semantic functions, including a role as a locative marker meaning 'front'/'in front of', and a temporal marker meaning 'next' (as in pii naa 'next year', literally 'year face/front'). There is a range of various 'face' expressions, in the noun + predicate pattern, in which naa 'face' is noun phrase head, with a following verbal modifier. 


\section{$156 \quad$ N. J. Enfield}

\subsection{1. naa-buut 'rotten-face'}

Elsewhere, buut means 'rotten', with reference especially to food which has gone bad, is repulsive, and no longer edible. The mood often attributed to someone with a 'rotten face' is khiat, which (as described above) involves brooding, directed non-involvement with someone who is the target of some ill-feeling. It would seem that the 'rotten-face' provides the khiat person with one way to explicitly show their ill-feeling (i.e. in the presence of the person towards whom they are khiat).

There is some latitude with regard to defining what exactly constitutes naa-buut 'rotten face'. An English speaker might call it a scowl or a sour face, but it seems to me best described as an anti-smile. By this I mean that a naa-buut 'rotten face' expression aims to look least like a smile as possible. A number of informants have defined naa-buut in just this way - as 'never smiling' - and accordingly, all speakers I consulted used the term naa-buut (or just buut alone) to describe this schematic illustration (the abstract "opposite" of the smile "(-)"):

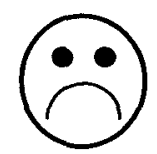

Note also that being naa-buut 'rotten-faced' does not entail an emotional state (such as khiat 'angry'), but may simply describe perceived ugliness or generally poor temperament (i.e. as a description of the kind of person who never smiles).

\subsection{2. naa-men 'smelly-face'}

The face which Lao speakers call 'smelly face' is essentially the classical "disgust face", with wrinkled nose, and raised upper lip. The element men 'smelly', however, refers not to a property of the face itself (in contrast to naa-buut 'rotten face', $\S 2.2 .1$., above), but to something smelly (i.e. rotten) which would cause someone to pull such a face. While naa-buut 'rotten face' is typically used to describe the face of someone who is of poor temperament, or who is khiat, or even caj-haaj ( $\$ 1.2$., above), the ex- 
pression naa-men 'smelly-face' would not be used to describe someone in such a mood. Rather, naa-men 'smelly-face' describes the face of a physically disgusted person only.

\subsection{3. naa-khùm 'furious-face'}

The term khùm refers to a kind of furious anger or rage, although certainly less strong than these English terms. To have a khùm face is to look like someone in such a state, typically manifest in classical signs of 'rage' in the face, such as exposed incisors, square mouth, frowning, and perhaps also exposed-whites-above-iris eyes (cf. § 2.4.1., below).

\subsection{4. naa-beq/naa-ñùq 'ready-to-cry face'}

The face of someone who is about to cry may be described as naa-beq or naa-ñùq, the latter term being less common, perhaps of regional origin. Neither beq nor ñùq have related meanings alone or in compound expressions other than these. The typical naa-beq includes a combination of slightly protruding, and perhaps trembling, lower lip, along with knitted and lowered eyebrows. The defining factor of naa-beq is evidently this lower lip pose (cf. sop-beq; § 2.3.2., below).

\subsection{The mouth}

There are a number of terms in Lao for facial expressions involving the mouth and parts of the mouth, mostly including the terms paak 'mouth', sop 'lips', and khèèw 'teeth'. It is worth noting, incidentally, that the term paak is only approximately translated as 'mouth' - it can be used to refer to the lips and outer part of the mouth as well, as in the expression paak tèek [mouth break] 'broken mouth' (referring to split or chapped lip(s)). 


\subsection{1. ñim 'smile'}

The term ñim 'smile' is apparently equivalent in meaning to English smile. The crucial idea is that the corners of the mouth are raised (and naturally this includes associated visible muscle movements around the eyes, cheeks, and jaw). In attributing an emotional state to a smiling person, Lao speakers generally describe them as dii-caj 'glad', and occasionally sabaaj(-caj) 'content' (if the smile in question is not judged to be a transient response to some specific happy stimulus).

\subsection{2. sop-beq 'about-to-cry lip'}

As described above ( $\$ 2.2 .4$.), someone who is about to cry may have a 'beq face' (naa-beq). This facial expression is localised in the lower lip, since it may also be described by the term sop-beq, i.e. having a 'beq lip'. The lower lip protrudes, typically trembling. In attributing an inner state to someone with this expression, most informants use the terms sia-caj 'sad', sook-saw 'unhappy', and occasionally bò-sabaaj 'uncomfortable, ill'. Most often the subject is said to be 'about to cry' (kamlang-ca-haj [progressive-irrealis-cry] or ca-haj-lèèw [irrealis-cry-perfective]).

\subsection{3. qaa-paak 'open-mouthed'}

The expression qaa-paak, referring to wide-openness of the mouth, is a verb + object construction somewhat like its English noun-incorporating counterpart open-mouthed. A doctor speaking Lao asks patients to qaapaak (where in English one would say open wide or say ' $a h$ '). The qaapaak 'open-mouthed' facial expression is not necessarily associated with emotion, since of course one may hold one's mouth wide open when at the doctor, when putting food in there, or in many other situations. When asked to attribute an emotional state to someone qaa-paak 'openmouthed', Lao speakers usually use the term tok-caj 'surprised'. Note that when one is qaa-paak 'open-mouthed', one cannot speak, a fact which would seem to be consistent with the idea of being 'surprised'. It would be unusual, if possible at all, to say laaw qaa-paak vaw [s/he speak openmouth] '?S/he spoke open-mouthed'. 


\subsection{4. ñom-paak 'clench-mouthed'}

The expression ñom-paak 'clench-mouthed' refers to tight compression of the lips, as in an expression of great determination. This may also be referred to as kat-khèèw 'biting-the-teeth'. When shown a picture of somebody with tightly compressed lips, informants used both terms ñompaak 'clench-mouthed' and kat-khèèw 'biting-the-teeth', and invariably attributed great determination to the subject, for example by saying that the person jaak het qan.daj.qan.nùng [want do something] 'wants to do something', or tang-caj [set.in.place-heart] 'intends (to do something), is determined'. Indeed, it would seem that the idea of 'clenching' is suggestive of readiness for springing into action. (One piece of related linguistic evidence is the four-syllable expression kat-khèèw-ñom-kon, literally 'bite-teeth-clench-bottom', which refers to a state of great determination and/or readiness for action.)

\subsection{Eyes and eyebrows}

There are a number of descriptions of facial expression in Lao involving taa 'eye(s)' and khiw 'eyebrow(s)'.

\subsection{1. taa-loo 'exposed-whites-above-iris eyes'}

The expression taa-loo (where loo does not occur independently) refers to wide-open eyes, where the whites of the eyes above the iris are visible. Informants most often describe someone with this expression as jaan 'afraid', especially when other aspects of the facial expression support (or at least do not contradict) this, for example if the subject is also qaa-paak 'open-mouthed' ( $\$ 2.3 .3$., above). If a subject combines taa-loo 'exposedwhites-above-iris eyes' with some incongruous pose, such as a smiling mouth, Lao informants attribute to the person a state of insanity ( $\mathrm{baa}$ 'insane'), or else describe them as a malevolent spirit (phii 'spirit, ghost', phii-baa [spirit-insane] 'lunatic'). 


\subsection{2. het taa-ñaj 'make big-eyes'}

To 'make big-eyes' - het taa-ñaj-is to open the eyes wide, with associated raising of the eyebrows. One who 'makes big eyes' does not necessarily expose the whites of their eyes above the iris. Most informants describe a face with taa-ñaj 'big eyes' as that of someone who is tok-caj 'surprised', although it may also mean that they are merely son-caj 'interested' in something.

\subsection{3. taa-tii 'eyes held open/apart'}

The term taa-tii has a similar meaning to taa-ñaj, but with a connotation of less control over the facial expression. The verb tii means 'hold an opening apart so as to provide access through', for example when holding a plastic bag open for someone to put something in. If you are taa-tii ' (with) eyes held open/apart', the connotation is that what you are seeing is so surprising or engaging that it 'holds your eyes open'.

\subsection{4. taa-khaang 'stuck-eyes'}

The term khaang conveys the idea of being 'stuck' or 'remaining, left over', as for example with reference to water which has not drained from a flat rooftop. To say that someone's eyes are khaang 'stuck' is to say that they are wide open and stuck there, i.e. that they do not close at all, not even momentarily for blinking. The open eyes of a corpse can be described as khaang, as well as the open eyes of someone sleeping or in a coma. When taa-khaang 'stuck eyes' is taken to be an index of some inner state, the idea is that the experiencer is so amazed or interested in what is happening in front of them that they cannot look away or even blink.

This points to what may be a general parameter in the description of facial expressions, namely the question of whether the pose is extended over time or not. The expression taa-khaang 'stuck-eyes' entails the pose being held over a stretch of time, while other terms describing facial expressions may make no such specification. Grin, for example, entails neither a momentaneous nor extended time profile. Thus, taa-khaang 'stuck-eyes' is not associated simply with being tok-caj 'surprised', but with this plus 
something more, which accounts for the persistence of the pose over time. For example, the person who is taa-khaang 'stuck-eyes' may be son-caj 'interested', or the like.

\subsection{5. ñik-khiw 'raise-eyebrows'}

While Lao has a basic transitive verb ñok 'to raise something', this verb cannot be used for raising one's eyebrows, unless the meaning intended is that the person physically pulls up their own eyebrows with the hands. To raise one's eyebrows as a facial gesture is referred to as nik-khiw (in the verb + object pattern, perhaps best literally glossed as 'raise-eyebrowed'). (The verb ñik does not occur elsewhere.) Informants have responded in a number of ways in attributing inner states to subjects with $\bar{n} i k$-khiw 'raised eyebrows', and it seems that accompanying features of facial expression are important factors in choosing between alternatives. Someone who is merely ñik-khiw 'with raised eyebrows', and without specific expression elsewhere in the face, may be described as son-caj 'interested', jaak-thaam 'wanting to question', or bò̀-sùa 'non-believing, doubtful'. The common theme here seems to be that the subject wants to know more about what is going on. When ñik-khiw 'raised eyebrows' is accompanied by taa-ñaj 'big eyes', taa-khaang 'stuck eyes', or especially taa-loo 'exposed-whitesabove-iris eyes', this is associated with being jaan 'afraid', as well as tokcaj 'surprised'.

\subsection{6. het khiw son 'making-eyebrows-collide'}

Lao does not have a term equivalent to English frown, and the idea of bringing one's eyebrows together is expressed as het khiw son 'make the eyebrows collide'. (The verb son is used elsewhere most notably for the butting of buffaloes' heads when they clash.) Inner states of son-caj 'interested', or kamlang-khit [progressive-think] 'thinking' are attributed to someone displaying this facial expression. 


\subsection{7. het khiw còòt-kan 'making-eyebrows-be-parked-together'}

The term het khiw còot kan means literally 'to make the eyebrows be parked together'. This is an alternative description of the 'knitted brow' just described as 'making the eyebrows collide' - the same comments apply.

\subsection{Comment}

The evidence presented in $\S 2$ has shown that Lao speakers' habitual ways of talking about facial expression differ from those of English speakers. It appears that for certain English expressions which seem to describe the face as a whole - such as frown or grimace - the Lao equivalents explicitly refer to relevant parts of the face, such as the eyebrows or the lips. Similarly, certain specific aspects of facial expression associated with certain specific emotions are singled out in Lao, such as for example the eyebrows, and the exposed-whites-above-iris eyes. Thus, it is not simply expressions on whole 'faces' that reveal and/or communicate feelings and thoughts, but rather expressions involving features or components of the face, such as the eyes, eyebrows, lips, mouth, or some combination of these. Certain descriptions of facial expressions make specific reference to components of the face, and I suggest that in further research, it would pay to consider facial expression as having a greater componentiality than is sometimes assumed. (Cf. Wierzbicka 1999, Chapter 4.)

\section{Conclusion}

While we may be led to attribute thoughts and/or feelings to a person on the basis of the expression on their face, it is not the case that a particular set of facial expressions conveys, either universally or specifically, a particular set of inner states or emotions. Firstly, there is no universal set of "emotions". What are denoted by words for 'emotions' are not actual phenomena, but conventional and emic descriptions of actual phenomena. Emotion terms denote concepts, by particular names in particular languages, such as angry, pissed off, or caj-haaj. When we work on the description of emotion, we cannot escape our reliance on language. Scientific inquiry is 
chronically linguocentric, and very often hopelessly monolinguocentric as well. (For these terms, see Enfield 2000.) It is unacceptable to adopt Ekman's (1973) perspective that '[r]egardless of the language, of whether the culture is Western or Eastern, industrialized or preliterate, ... facial expressions are labeled with the same emotion terms: happiness, sadness, anger, fear, disgust, and surprise' (Ekman 1973: 220). It is simply not true that in all societies these labels are applied, because these labels are English, and not all societies know and/or use English.

It is interesting to consider the parallel between research on facial expression of emotion, and research on the semantics of colour terms. (See Foley 1997: Ch. 7 for a useful recent review of colour research.) It has been argued (e.g. by Lucy 1996 and Wierzbicka 1996) that colour terms are not labels for mathematically defined frequency bandwidths, but are labels for concepts, which of course may in turn relate to, or be derived from, those natural phenomena. Research on colour semantics has been traditionally fraught with problems of translation, and the unquestioned assumption that the biological basis of the 'meaning' intended by a colour term has provided researchers with a way of anchoring language in uncontroversial human universals. But the problem lies in the very assumption that these universal biological phenomena - such as visual perceptual apparatus (in the case of colour) or empirically definable visceral responses (in the case of "emotion") - are what linguistic expressions actually label. There is no evidence that they are. ${ }^{4}$

The data presented here supports a view that different ethnolinguistic systems analyse the expressive face using somewhat different conventions. Universals of thought and feeling undoubtedly exist, along with ethologically grounded explanations for visceral phenomena and universal aspects of facial behaviour. But these have no direct link to what we refer to, by lexical items in natural languages, as emotions. We cannot take "basic emotions" for granted, and certainly not as something that pre-defined facial expressions (if these could be identified) universally signify. And it is essential to take an empirical and maximally assumption-free approach to understanding what "facial expressions" really consist of, cross-culturally and cross-linguistically. Hence the value of "folk" analyses of the data, such as I have described here. I therefore reiterate Russell's recent call for more empirical data on the possible interpretations of facial expression which natural languages reveal. 


\section{Notes}

1. Transcription of Lao is a simplified system, with tone-marking omitted. Note that the consonant symbol ' $j$ ' in this system (as in the IPA system) is pronounced like English ' $y$ ' in you and boy; 'c' is pronounced in a similar manner to English ' $\mathrm{j}$ ' in Jill; ' $q$ ' represents a glottal stop. Symbols 'ù', 'ò', 'è' represent high-back-unrounded, low-back, and low-front vowels, respectively. All data are from fieldnotes, and from field interviews conducted in Sisavat and Saphang Mo villages in Vientiane, Lao PDR, January-May, and July-August 1998. Syban Khoukham's assistance is gratefully acknowledged. Pitsana Vayaphanh also provided generous consultation, and Sotaro Kita gave useful comments on a late version of the text.

2. Disgust is a particularly physical emotion, with a genuine functional/biological aspect to it, and one could imagine that certain things - such as, say, rotten food - would evoke disgust in anyone with a human body, in any cultural sphere. But it is interesting to note that culture can differently define what disgusts people in this physical way. Thus, one can easily find everyday practices which are embraced by one group, yet physically revolting for another. Rural Lao people enthusiastically snack on insects of different kinds (crickets, grasshoppers, beetles), a practice which the average urban Australian, say, would find physically disgusting. On the other hand, the popular practice of oral sex in Anglo culture is regarded similarly as physically disgusting in many other settings (e.g. rural Burma, Spiro 1977: 232). These fascinating culture-specific visceral responses, showing the influence that culture may have on our biological responses, are worth closer and broader research.

3. Tùùn 'awaken' has an interesting argument structure, being $\mathrm{S}=\mathrm{A}$ ambitransitive, where the subject is semantically a patient; thus, cf. khòò tùùn hok moong [I awaken six o'clock] 'I woke up at six o'clock', khòoj tùùn caw [I awaken you] 'I (got a) start (from) you'/'You gave me a start'. In the second example, the syntactic object caw 'you' is the stimulus for the event of surprise predicated by tùùn 'awaken' of its subject khòoj ' $\mathrm{I}$ '.

4. Even so, colour research has resulted in interesting findings, such as the various implicational statements which Berlin and Kay (1969) originally put forward. While there are serious methodological problems with their research (Lucy 1996), it is nevertheless interesting to consider the kinds of questions being asked. If a language has only three "basic colour terms" (as Berlin and Kay define these), can we predict what will they be? Berlin and Kay argued in the affirmative for LIGHT ("white'), WARM ('red'), and DARK/COOL ('black/blue/green'). If a language had five terms, they argued, then 'yellow' would appear, along with a split of DARK/COOL into 'black', and 'green/blue'. Could we follow such a line with respect to facial expressions? If a language had only one "basic facial expression term", would it be "smile'? (Cf. Wierzbicka 1999: 275, 282.) But this may be the wrong kind of question to ask (cf. Lucy 1996 on colour). 


\section{References}

Berlin, Brent, and Paul Kay

1969 Basic Color Terms. Berkeley: University of Califomia Press.

Diller, Anthony V. N., and Preecha Juntanamalaga

1990 'Full hearts' and empty pronominals in Thai. Australian Journal of Linguistics 10 (2): 231-256.

Ekman, Paul 1973

Darwin and Facial Expression: A Century of Research in Review. New York: Academic Press.

1992 An argument for basic emotions. Cognition and Emotion. 6 (3/4): $169-200$.

Enfield, N. J.

2000 On linguocentrism. In: Martin Pïtz and Marjolijn H. Verspoor (eds.), Explorations in Linguistic Relativity, 125-157. Amsterdam: John Benjamins.

2001 Semantic analysis of body parts in emotion terminology: avoiding the exoticisms of "obstinate monosemy" and "online extension". Pragmatics and Cognition 9.2 .

Foley, William A.

1997 Anthropological Linguistics. London: Blackwell.

Goddard, Cliff 1997

Contrastive semantics and cultural psychology: 'Surprise' in Malay and English. Culture and Psychology 3 (2): 153-181.

Jaisser, Annie

1990 DeLIVERing an introduction to psycho-collocations with SIAB in White Hmong. Linguistics of the Tibeto-Burman area 13 (1): 159-177.

Lucy, John

1996 The linguistics of 'color'. In: C. L. Hardin and Luisa Maffi (eds.), Color Categories in Thought and Language, 320-346. Cambridge: Cambridge University Press.

Matisoff, James A.

1986 Hearts and minds in Southeast Asian languages and English: an essay in the comparative lexical semantics of psycho-collocations. Cahiers de Linguistique Asie Orientale 15(1): 5-57.

Oey, Eric. M.

1990 'Psycho-collocations' in Malay: a Southeast Asian areal feature. Linguistics of the Tibeto-Burman Area 13(1): 141-158.

Russell, James A.

1995 Facial expressions of emotion: What lies beyond minimal universality? Psychological Bulletin 118 (3): 379-391. 


\section{$166 \quad$ N. J. Enfield}

Spiro, Melford E.

1977 Kinship and Marriage in Burma. Berkeley: University of California Press.

Wierzbicka, Anna

1996 Semantics: Primes and Universals. Oxford: Oxford University Press.

1999 Emotions across Languages and Cultures: Diversity and Universals. Cambridge: Cambridge University Press.

Zajonc, R. B.

$1980 \quad$ Feeling and thinking. Preferences need no inferences. American Psychologist 35(2): 151-175.

1984 On the primacy of affect. American Psychologist 39 (2): 117-123. 